\title{
Routine preoperative echocardiograms in select patients with adolescent idiopathic scoliosis rarely show abnormalities and do not predict adverse cardiovascular events
}

\begin{abstract}
Study Design: Retrospective

Objectives: The prevalence and clinical significance of cardiac abnormalities detected on ECHO in surgically treated AIS patients is unclear. Our goals were to determine the prevalence of cardiac abnormalities detected on echocardiography (ECHO) in patients undergoing surgery for adolescent idiopathic scoliosis (AIS). Further, we aimed to determine whether preoperative curve magnitude is associated with abnormal ECHO findings and whether ECHO abnormalities are associated with perioperative cardiovascular complications.

Methods: We reviewed clinical records of 619 patients aged 10 to 18 years with AIS who underwent spinal fusion from 2000 through 2015. Indications for ECHO were a major curve greater than $70^{\circ}$ or recommendation by primary care providers. Major curve magnitudes were calculated using Cobb angles. We assessed for adverse perioperative cardiovascular events. Statistical significance was tested using Student $t$ test with an alpha level of $<.05$.

Results: Forty-three (7\%) patients had a preoperative ECHO within 6 months before surgery (23 normal ECHOs, 16 normal variants (including trivial valvular abnormalities), and 4 abnormal ECHOs (all mild functional or structural abnormalities). There was no association between presence of cardiac abnormalities and major curve magnitude ( $\mathrm{p}=$ .38). There were no documented perioperative adverse cardiovascular events in any of the 43 cases nor in the 576 patients who did not have preoperative ECHOs.

Conclusion: Patients with abnormal ECHOs did not experience perioperative cardiovascular events. Curve magnitude was not associated with abnormal ECHO findings. Routine preoperative ECHOs in AIS patients based on curve magnitude did not change management in these patients.
\end{abstract}

Keywords: Adolescent idiopathic scoliosis, Adverse cardiovascular events, Echocardiograms, Preoperative evaluation, Spinal fusion
Volume 7 Issue I - 2017

Sandesh S Rao,' Amit Jain,' David Zhou,' Dolores B Njoku, ${ }^{2}$ Paul D Sponseller'

'Department of Orthopaedic Surgery, The Johns Hopkins University, USA

${ }^{2}$ Departments of Anesthesia and Critical Care Medicine and Pediatrics, Johns Hopkins Medical Institutions, USA

Correspondence: Sandesh S Rao, MD, Department of Orthopaedic Surgery, The Johns Hopkins University, 60I N Caroline St, Floor 5, Baltimore, MD 2 I205, USA, Tel 30I-7583298, Fax 410-955-3137, Email sraol6@jhmi.edu

Received: October 27, 2016 | Published: January 06, 2017

\section{Introduction}

Scoliosis affects approximately $2 \%$ to $3 \% 0^{1,2}$ of the population, with most diagnoses occurring before 15 years of age. More than twothirds of cases are attributed to idiopathic causes, and the remaining one-third are attributed to underlying abnormalities in which systemic comorbid conditions may exist. ${ }^{3,4}$ Cardiac abnormalities represent a group of comorbid conditions in patients with scoliosis, including those with adolescent idiopathic scoliosis (AIS). ${ }^{5-8}$

Cardiac abnormalities are especially relevant when considering surgical management for these patients. The presence of a cardiac abnormality is not a contraindication to surgical scoliosis correction ${ }^{9,11}$ but is important to understand the nature and severity of the abnormality to assess operative risk and optimize patients from a medical and surgical standpoint. ${ }^{9-11}$

Studies have reported cardiac valvular abnormalities, including mitral valve prolapse, in patients with AIS. ${ }^{5,6,12,13}$ The prevalence of cardiac valvular abnormalities, and specifically mitral valve prolapse, is higher in patients with a strong family history of scoliosis ${ }^{6}$ or with other known comorbidities, including anorexia nervosa, psoriasis, cystic fibrosis, and spondylolisthesis. ${ }^{5}$ Ipp et al. ${ }^{13}$ reported a higher prevalence of aortic root and aortic valve abnormalities in patients with scoliosis. However, there has been limited discussion in these studies with respect to ventricular function and no reports correlating cardiac abnormalities with adverse events.

Various recommendations for presurgical screening with an echocardiogram (ECHO) have been proposed. The current recommendation from the American Society of Anesthesiologists Task Force on Preanesthesia Evaluation ${ }^{14}$ is to pursue preoperative cardiac evaluation (including ECHO) in noncardiac surgery only when there is substantial concern based on history and physical examination. This recommendation is generalized to all preoperative noncardiac surgery evaluations and is not specific for children with AIS undergoing scoliosis correction. In patients with AIS, there is concern for undiagnosed and underlying cardiac abnormalities that may increase operative risk. ${ }^{3,5-7,9-11,13}$

Our goals were to determine (1) the prevalence of ECHO-detected cardiac abnormalities, (2) whether preoperative curve magnitude is associated with abnormal ECHO findings, and (3) whether an association exists between perioperative cardiovascular complications and ECHO abnormalities. We hypothesized that structural cardiac abnormalities shown on preoperative ECHO in patients with AIS 
would be associated with perioperative adverse cardiovascular events. Additionally, we predicted that spinal curve magnitude would be associated with the presence of cardiac abnormalities.

\section{Materials and methods}

Clinical records of all 619 children with AIS who underwent spinal fusion surgery from 2000 through 2015 by a single surgeon at a major academic referral center were reviewed. We included patients aged 10-18 years who underwent a preoperative ECHO within 6 months before surgery. ECHO was performed at the discretion of the surgeon or primary care providers. Indications included major curve angles greater than $70^{\circ}$ or for preoperative risk assessment. Patients who received ECHO assessments for prior history of cardiac surgery, congenital cardiac disease, murmur auscultated on cardiac examination, concern for Marfan syndrome, prior cardiotoxic chemotherapeutic exposure, and evaluation for hypertension and tachycardia were excluded from the study. If more than one ECHO was obtained, the study closest to the date of surgery was used. Institutional review board approval was obtained for this retrospective cohort study.

Data collected for each patient consisted of age at the time of surgery, sex, diagnosis, severity of spinal curvature, date of ECHO, date of surgery, and ECHO results. All ECHOs were interpreted by one of several pediatric cardiologists. Results of the ECHO included assessment of all four cardiac valves and chambers, aortic root and aorta, pulmonary vein and artery, and any abnormalities (including pericardial effusion, septal abnormalities, patent ductus arteriosus, and atrial and ventricular septal defect).

Patients with left ventricular (LV) pathologies, including systolic and diastolic dysfunction, and right ventricular (RV) pathologies were defined as having anatomical or wall motion abnormalities or changes in cardiac pressures or output. Valvular abnormalities were defined on the basis of established standard values for stenosis and regurgitation. ${ }^{15,16}$ Severity of cardiac abnormalities was determined by a pediatric cardiologist.

ECHO results were initially reviewed by a pediatric cardiologist. This report was then reviewed by a pediatric anesthesiologist for surgical relevance. All ECHO reviewers were blinded to the presence or absence of adverse events.

Additionally, all 619 patient records were evaluated by a pediatric orthopedic surgeon and pediatric anesthesiologist to assess for adverse perioperative cardiovascular events by reviewing operative notes and discharge summaries from the hospitalization. Adverse cardiovascular events were defined as cardiac arrest, cardiac dysfunction or development of an arrhythmia requiring intervention. Patients with sinus tachycardia were excluded from this definition.

\section{Statistical analysis}

We calculated means, ranges, and standard deviations for patient characteristics and spinal curvature. Statistical significance was tested using Student $t$ test, and results of $\mathrm{p}<.05$ were considered significant.

\section{Results}

Of the 619 patients who underwent surgery, 43 (7\%) had preoperative ECHOs within 6 months before surgery. Among these 43 patients (29 girls), the mean age was 14.2 (range 10.8-17.7) years (Table 1).
Table I Characteristics of 619 patients who underwent surgical correction of adolescent idiopathic scoliosis, by preoperative echocardiogram status

\begin{tabular}{|c|c|c|c|c|}
\hline & $\begin{array}{l}\text { No ECHO } \\
(n=576)\end{array}$ & & $\begin{array}{l}\text { Received ECHO } \\
(n=43)\end{array}$ & \\
\hline Characteristic & Mean (SD) & n (\%) & Mean (SD) n (\%) & $\mathbf{p}$ \\
\hline Age at surgery (y) & $14.6 \pm 1.70$ & & $14.2 \pm 2.02$ & 0.14 \\
\hline Female sex & & $443(77)$ & $29(66)$ & 0.16 \\
\hline Major curve angle $\left({ }^{\circ}\right)$ & $59.1 \pm 11.5$ & & $76.7 \pm 17.1$ & $<.05$ \\
\hline
\end{tabular}

\section{ECHO: Echocardiogram; SD: Standard Deviation}

Thirty-nine ECHOs were read as functionally normal (herein, "normal"), and four were read as abnormal. (In the normal group, 16 ECHOs were read as normal variants, which included trivial or mild valvular abnormalities. These valvular abnormalities were deemed to be within the range of normal ECHO findings ${ }^{17-19}$ because of a lack of associated clinical symptomotology and severity on ECHO.) Patient characteristics, including age, sex, and major curve magnitude did not differ significantly between the normal and abnormal groups (Table 2).

Table 2 Characteristics of 43 patients with adolescent idiopathic scoliosis, stratified by echocardiogram results

\begin{tabular}{lllll}
\hline & $\begin{array}{l}\text { Normal ECHO } \\
(\mathbf{n}=\mathbf{3 9})\end{array}$ & $\begin{array}{l}\text { Abnormal ECHO } \\
(\mathbf{n}=\mathbf{4})\end{array}$ & \\
Characteristic & $\begin{array}{l}\text { Mean (SD) } \\
\text { n (\%) }\end{array}$ & $\begin{array}{l}\text { Mean (SD) } \\
\text { n (\%) }\end{array}$ & p \\
\hline Age at surgery $(y)$ & $14.2 \pm 2.0$ & $14.6 \pm 2.81$ & .70 \\
Female sex & & $27(68)$ & $2(50)$ & .45 \\
Major curve angle $\left(^{\circ}\right)$ & $77.5 \pm 16.9$ & $69.5 \pm 19.7$ & .38
\end{tabular}

\section{ECHO: Echocardiogram}

There were no documented perioperative adverse cardiovascular events in any of the 43 cases. Among the 576 patients who did not undergo an $\mathrm{ECHO}$, one developed trigeminy during postoperative hospitalization in which he was hemodynamically stable, did not require intervention, and experienced spontaneous resolution. Additionally, the episode did not lengthen the patient's hospital stay or alter his discharge status.

\section{Discussion}

In patients with AIS without other comorbidities or concerning signs and symptoms, we hypothesized that ECHOs would still show structural cardiac abnormalities and that these abnormalities would be associated with adverse cardiovascular events. Only a small percentage of the population that received ECHOs showed evidence of cardiac structural abnormality on imaging. Our findings add to those of previous studies documenting cardiac abnormalities in patients with AIS..$^{5,6,12,13,20,21}$ Irrespective of ECHO findings, there were no documented adverse perioperative cardiovascular events. We also found no correlation between the major spinal curve magnitude and presence of cardiac abnormalities. Currently, there is no consensus on the use of preoperative ECHOs in patients with AIS..$^{5,6,13,22}$ Our findings disprove our original hypothesis and suggest that the practice of obtaining preoperative ECHOs for patients with AIS may be unnecessary in the absence of clinical signs or symptoms.

Seven percent of patients had evidence of structural abnormalities on ECHO, all of whom had some component of LV pathology, including three patients with specific LV dysfunction and one patient with a dysplastic aortic valve. Of note, Colomina et al. ${ }^{5}$ reported zero of 29 patients with ventricular abnormality. Additionally, Ipp et al. ${ }^{13}$ reported only one of 212 patients with LV abnormality. Although not 
a common finding in these previous studies, Huh et al. and Li et al specifically found abnormalities in left ventricular function in patients with AIS. ${ }^{20,21}$ However, these studies also found LV dysfunction to be associated with more severe curves, which we did not find in our study population. Additionally, these studies did not evaluate their findings with any immediate clinical endpoint with respect to corrective surgery. Studies of adults with LV diastolic dysfunction have shown trends toward longer hospital stays. ${ }^{23,24}$ Irrespective of long-term repercussions, in our study, despite evidence of LV pathology, patients did not have a delay in time to surgery and did not experience cardiovascular perioperative events.

Studies have found evidence of RV dysfunction and valvular abnormalities in patients with AIS..$^{5-8,12,13}$ Dhuper et al. ${ }^{12}$ and Colomina et al. $^{5}$ commented on the prevalence of mitral valve prolapse. In comparison with their control populations, they noted mitral valve prolapse to occur at four times the rate in patients with AIS.,12 However, prior data has shown no higher perioperative risk with the finding of mitral valve prolapse in asymptomatic patients. ${ }^{25} \mathrm{Li}$ et al. ${ }^{22}$ showed a significant decrease in RV ejection fraction in patients with severe scoliosis (major curve angles $>80^{\circ}$ ) using the tricuspid annular plane systolic excursion as a surrogate for ejection fraction. Additionally, perioperative RV dysfunction has been associated with increased rates of morbidity and mortality in cardiac and noncardiac surgery. ${ }^{26-30}$ However, in our study, there was no evidence of valvular abnormalities beyond what would be considered within the normal variation in the pediatric population. ${ }^{15-17} \mathrm{RV}$ dysfunction was also not present in any of the ECHOs, and tricuspid annular plane systolic excursion (TAPSE) values were within normal parameters.

On the basis of our study and several others, ${ }^{5-8}$ patients with AIS have an increased incidence of cardiac abnormalities on ECHO. Because all of our patients were asymptomatic, these abnormalities appear to be structural rather than functional. Additionally, cardiac abnormalities were not associated with adverse cardiovascular events.

There is no consensus on the value of perioperative ECHOs in patients with AIS, even in those with prior cardiac abnormalities or concerning histories. The issue of value is pertinent in the era of "high-value care," focused on minimizing unnecessary testing and costs that will not change clinical management. The professional fee at our institution for obtaining an ECHO was \$258 US and represents a potentially unnecessary cost that did not affect clinical management. On the basis of our single-center, single-surgeon results, echocardiographic abnormalities in patients with AIS do not appear to be associated with perioperative adverse cardiovascular events, even in those with prior history of cardiac abnormalities. Irrespective of whether patients underwent ECHO, the absence of malignant arrhythmia and only one documented case of an arrhythmia points to such events being rare in this population as a whole.

A strength of our study is the assessment of a large number of patients with AIS who underwent surgical correction of scoliosis. Our study is limited by its retrospective, observational nature. Additionally, ECHOs were reviewed and reported by several pediatric cardiologists and not standardized to a single reader. Finally, initial indication for an $\mathrm{ECHO}$ was a major curve angle greater than $70^{\circ}$; however, several patients with major curve angles less than this underwent ECHO.

We suggest that the practice of obtaining a preoperative ECHO for patients with AIS may need to be re-evaluated irrespective of severity of curvature. Cost-benefit ratios should be considered during preoperative evaluation in light of history and physical examination to determine if the patient might benefit from a preoperative ECHO. Our study did not detect any ECHO findings that may delay time to surgery and thus findings did not change perioperative patient management in any single patient. Given the findings of this study routine preoperative ECHOs in select patients with AIS including those with cobb angles greater than 70 do not benefit from preoperative cardiac evaluation by ECHO.

\section{Acknowledgments}

None.

\section{Conflicts of interest}

None.

\section{References}

1. Herring JA. Tachdjian's Pediatric Orthopaedics. WB Saunders, Philadelphia, USA. 2002.

2. Wise CA, Gao X, Shoemaker S, et al. Understanding genetic factors in idiopathic scoliosis, a complex disease of childhood. Curr Genomics. 2008;9(1):51-59.

3. Janicki JA, Alman B. Scoliosis: Review of diagnosis and treatment. Paediatr Child Health. 2007;12(9):771-776.

4. Rosenberg JJ. Scoliosis. Pediatr Rev. 201132():397-398.

5. Colomina MJ, Puig L, Godet C, et al. Prevalence of asymptomatic cardiac valve anomalies in idiopathic scoliosis. Pediatr Cardiol. 2002;23(4):426-429.

6. Hirschfeld SS, Rudner C, Nash CL, et al. Incidence of mitral valve prolapse in adolescent scoliosis and thoracic hypokyphosis. Pediatrics. $1982 ; 70(3): 451-454$.

7. Liu L, Xiu P, Li Q, et al. Prevalence of cardiac dysfunction and abnormalities in patients with adolescent idiopathic scoliosis requiring surgery. Orthopedics. 201033(12):882

8. Primiano FP, Nussbaum E, Hirschfeld SS, et al. Early echocardiographic and pulmonary function findings in idiopathic scoliosis. $J$ Pediatr Orthop. 1983;3(4):475-481.

9. Coran DL, Rodgers WB, Keane JF, et al. Spinal fusion in patients with congenital heart disease. Predictors of outcome. Clin Orthop Relat Res. 1999364:99-107.

10. Kadhim M, Spurrier E, Thacker D, et al. Scoliosis surgery in children with congenital heart disease. Spine (Phila Pa 1976). 2014;39:E211E218.

11. Perez-Caballero C, Sobrino E, Vazquez JL, et al. Complication of surgery for scoliosis in children with surgically corrected congenital cardiac malformations. Cardiol Young. 2009;19(3):272-277.

12. Dhuper S, Ehlers KH, Fatica NS, et al. Incidence and risk factors for mitral valve prolapse in severe adolescent idiopathic scoliosis. Pediatr Cardiol. 199718(6):425-428.

13. Ipp L, Flynn P, Blanco J, et al. The findings of preoperative cardiac screening studies in adolescent idiopathic scoliosis. J Pediatr Orthop. 2011;31(7):764-756.

14. Apfelbaum JL, Connis RT, Nickinovich DG, et al. Practice advisory for preanesthesia evaluation: an updated report by the American Society of Anesthesiologists Task Force on Preanesthesia Evaluation. Anesthesiology. 2012116:522-538.

15. Baumgartner H, Hung J, Bermejo J, et al. Echocardiographic assessment of valve stenosis: EAE/ASE recommendations for clinical practice. Eur J Echocardiogr. 2009;10(1):1-25.

16. Grattan MJ, Mertens L. Echocardiographic assessment of ventricular function in pediatric patients: a comprehensive guide. Future Cardiol. 2014;10(4):511-523. 
17. Biancaniello T. Innocent murmurs. Circulation. 2005;111:e20-e22.

18. Brand A, Dollberg S, Keren A. The prevalence of valvular regurgitation in children with structurally normal hearts: a color Doppler echocardiographic study. Am Heart J. 1992;123(1):177-180.

19. Ratti C, Grassi L, De Maria E, et al. Cardiac auscultation in children. Recenti Prog Med. 2014;105(12):469-472.

20. Huh S, Eun LY, Kim NK, et al. Cardiopulmonary function and scoliosis severity in idiopathic scoliosis children. Korean J Pediatr. 201558(6):218-223.

21. Li S, Yang J, Zhu L, et al. Left ventricular mechanics assessed by two-dimensional speckle tracking echocardiography in children and adolescents with idiopathic scoliosis. Clin Spine Surg. 2016.

22. Li S, Yang J, Li Y, et al. Right ventricular function impaired in children and adolescents with severe idiopathic scoliosis. Scoliosis. 2013;8:1.

23. Cho DH, Park SM, Kim MN, et al. Presence of preoperative diastolic dysfunction predicts postoperative pulmonary edema and cardiovascular complications in patients undergoing noncardiac surgery. Echocardiography. 2014;31(1):42-49.
24. Xu-Cai YO, Brotman DJ, Phillips CO, et al. Outcomes of patients with stable heart failure undergoing elective noncardiac surgery. Mayo Clin Proc. 2008;83(3):280-238.

25. van Klei WA, Kalkman CJ, Tolsma M, et al. Pre-operative detection of valvular heart disease by anaesthetists. Anaesthesia. 2006;61(2):127-132.

26. Denault AY, Haddad F, Jacobsohn E, et al. Perioperative right ventricular dysfunction. Curr Opin Anaesthesiol. 2013;26(1):71-81.

27. Fox DL, Stream AR, Bull T. Perioperative management of the patient with pulmonary hypertension. Semin Cardiothorac Vasc Anesth. 2014;18(4):310-318

28. Hammill BG, Curtis LH, Bennett-Guerrero E, et al. Impact of heart failure on patients undergoing major noncardiac surgery. Anesthesiology. 2008;108(4):559-567.

29. McCurry KR, Keenan RJ. Controlling perioperative morbidity and mortality after lung transplantation for pulmonary hypertension. Semin Thorac Cardiovasc Surg. 1998;10(2):139-143.

30. Zarbock A, Van Aken H, Schmidt C. Management of right ventricular dysfunction in the perioperative setting. Curr Opin Anaesthesiol. 2014;27(4):388-393. 